\title{
Decorin reduces hypertrophic scarring through inhibition of the TGF-ß1/Smad signaling pathway in a rat osteomyelitis model
}

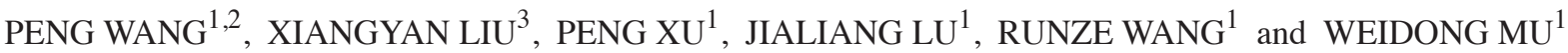 \\ ${ }^{1}$ Department of Traumatic Orthopaedics, Shandong Provincial Hospital Affiliated to Shandong University, Jinan, \\ Shandong 250012; ${ }^{2}$ Department of Orthopaedics, Weihai Municipal Hospital, Weihai, Shandong 264200; \\ ${ }^{3}$ Department of Thoracic Surgery, Shandong Provincial Hospital Affiliated to Shandong University, \\ Jinan, Shandong 250012, P.R. China
}

Received May 12, 2015; Accepted June 27, 2016

DOI: $10.3892 /$ etm.2016.3591

\begin{abstract}
Chronic osteomyelitis is a bone infection that results in hypertrophic scarring of the soft tissue surrounding the infected bone. This scarring can create functional problems and its treatment is challenging. The aim of the present study was to evaluate the efficacy of decorin in treating scar formation in osteomyelitis and the underlying mechanism of its action. A rat osteomyelitis model was used, and animals were divided into three groups, as follows: Group A (control), group B (osteomyelitis model) and group C (decorin-treated). $\mathrm{X}$-ray scans, hematoxylin and eosin (H\&E) staining and Masson's trichrome staining were performed to observe changes in femur and muscle tissue. In order to assess the role of the transforming growth factor $\beta 1$ (TGF- $\beta 1) / \mathrm{Smad}$ signaling pathway in scar formation in osteomyelitis, alterations in muscle tissue morphology and in the activation of key members of the TGF- $\beta 1 / \mathrm{Smad}$ signaling pathway were investigated in groups A and B. According to the results of H\&E staining, evident fibrosis in muscle tissue were observed at days 14 and 28 in group B. Simultaneously, the expression levels of key members of the TGF- $\beta 1 /$ Smad signaling pathway were increased. Subsequent to treatment with decorin in group C, scarring was reduced, and significant downregulation of collagen I, TGF- $\beta 1$, phosphorylated (p)Smad 2 and pSmad3 protein expression levels was observed at days 14 and 28 compared with the osteomyelitis group. In conclusion, these results suggest that activation of TGF- $\beta 1$ may serve an important role in the formation of scars in osteomyelitis and that decorin can reduce scar formation in an osteomyelitis rat model through inhibition of the TGF- $\beta 1 / \mathrm{Smad}$ signaling pathway.
\end{abstract}

Correspondence to: Dr Weidong $\mathrm{Mu}$, Department of Traumatic Orthopaedics, Shandong Provincial Hospital Affiliated to Shandong University, 324 Jing Wu Road, Jinan, Shandong 250012, P.R. China E-mail: sdphweidongmu@126.com

Key words: osteomyelitis, scar, transforming growth factor- $\beta 1$, Smad, decorin

\section{Introduction}

Chronic osteomyelitis is a complication resulting from trauma, which involves the combination of disruption of the blood supply to the bone and soft tissue destruction (1). The treatment of chronic osteomyelitis is challenging, due to prolonged therapy and recurrence (2). Debridement and antibiotic therapy are common treatments for osteomyelitis. In recent years, the Ilizarov method involving radical excision of the infected bone and distraction osteogenesis with bone transport has shown promising results in the treatment of chronic osteomyelitis $(3,4)$. However, scars occurring in chronic osteomyelitis can result in deformities and restriction of the mobility of joints and extremities. The pathogenesis of hypertrophic scarring is not fully understood, and clinical treatment has yet to be clearly defined.

Hypertrophic scarring mainly results from disorders of the collagen metabolism (5). Type I collagen is the major fibrous collagen, which is regulated by the transforming growth factor $\beta$ (TGF- $\beta$ ) signaling pathway at the translational level (6). Three isoforms of TGF- $\beta$ have been identified in mammals, including TGF- $\beta 1$, TGF- $\beta 2$ and TGF- $\beta 3$. Active TGF- $\beta 1$ is a major cytokine that stimulates the gene transcription of type I collagen (7). Therefore, blocking the TGF- $\beta 1$ signaling pathway may theoretically suppress the production of collagen I, thus preventing the formation of hypertrophic scars.

Decorin is a natural inhibitor of TGF- $\beta 1$, which has been demonstrated to have a beneficial effect of anti-fibrosis in hypertrophic scars formed after burns (8). However, to the best of our knowledge, no studies have investigated the effect of decorin on hypertrophic scars in chronic osteomyelitis. Thus, the present study was designed to investigate the in vivo effect of decorin in an osteomyelitis rat model. It was hypothesized that the TGF- $\beta 1$ cascade is involved in scar formation, and decorin was found to have an inhibitory effect on scarring in osteomyelitis through the TGF- $\beta 1$ signaling pathway.

\section{Materials and methods}

Treatment groups and treatment of animal model. A total of 72 Wistar rats 3 months of age with a weight of 300-350 g were provided by the Animal Center of Shandong University (Jinan, 
China). The study was approved by the Ethics Committee of Shandong Provincial Hospital Affiliated to Shandong University. The animals were kept and fed under standard conditions of temperature $\left(22 \pm 2^{\circ} \mathrm{C}\right.$, humidity $(45 \pm 5) \%$ and $12 \mathrm{~h}$ light.. Animals were randomly divided into three groups: Group A, control rats; group B, osteomyelitis model rats; and group $\mathrm{C}$, decorin-treated osteomyelitis rats.

Animals were given an intraperitoneal injection of 90 mg/kg 10\% ketamine (Jiangsu Hengrui Medicine Co., Ltd., Lianyungang, China) to anesthetize for $30 \mathrm{~min}$. The left leg of the rats was shaved and disinfected, and soft tissue was dissected from the lateral region of the femur. In group A (control group), the femur was exposed and subsequently the wound was sutured, with no further intervention. In group B, the chronic osteomyelitis model was established according to the model described by Inanmaz et al (9). The fracture of the femur was performed using vascular forceps and was fixed by Kirschner wire through the bone cavity. A saline solution containing $10^{6}$ colony-forming units $/ \mathrm{ml}$ methicillin-sensitive Staphylococcus aureus (MSSA; Nanjing Bianzhen Biotechnology Co., Ltd., Nanjing, China) was injected into the medullary cavity for bacterial inoculation, and the skin was then closed and disinfected. In group $\mathrm{C}$ (decorin-treated group), the animals were treated with decorin subsequent to the bacterial inoculation in osteomyelitis model rats. Recombinant mouse decorin was purchased from R\&D Systems Inc. (Minneapolis, MN, USA), and a dose of $50 \mu \mathrm{g}$ was injected in the muscle tissue surrounding the fracture on post-wounding days 0,1 , and 2 . Subsequent to the surgery, the animals were closely followed, and their weight and temperature were monitored regularly.

Groups A and B were designed to examine the formation of hypertrophic scarring in osteomyelitis and to assess the involvement of TGF- $\beta 1$ signaling in scar formation. Muscle tissues from these groups were harvested on days 7, 14 and 28 after surgery. Group $\mathrm{C}$ was designed to evaluate the efficacy and mechanism of decorin in treating scars formed as a result of osteomyelitis. Muscle tissues in group $\mathrm{C}$ were harvested on days 14 and 28 after surgery.

At the designated times, the animals were sacrificed by decapitation following the intraperitoneal injection of $90 \mathrm{mg} / \mathrm{kg}$ $10 \%$ ketamine, and muscle tissues surrounding the femur were harvested. The tissues were washed in normal saline and bisected. One of the two tissue sections was fixed in $4 \%$ formaldehyde for hematoxylin-eosin (H\&E) or Masson's trichrome staining. The other part of each tissue was snap-frozen in liquid nitrogen and stored at $-80^{\circ} \mathrm{C}$ for immunoblotting.

Radiography. Radiographs of the femur were obtained on post-wounding days 7, 14 and 28 in order to evaluate alterations of osteomyelitis, such as periosteal reaction, sclerosis and osteolysis (10).

Histological analysis. H\&E and Masson's trichrome staining were performed to examine the fibrosis of muscles. Tissue samples were fixed in $4 \%$ formaldehyde for $24 \mathrm{~h}$. Routine dehydration, paraffin embedding and serial sectioning into $5-\mathrm{mm}$ samples were sequentially performed. Subsequent to staining using H\&E or Masson's trichrome, the sections were mounted and observed for histology under a light microscope.
Immunoblotting analysis. The protein expression levels of TGF- $\beta 1$, TGF- $\beta$ receptor I (T $\beta \mathrm{RI}), \mathrm{T} \beta \mathrm{RII}$, phosphorylated-Smad2 (pSmad2), pSmad3, Smad2/3 and type I collagen were determined by western blot analysis. Muscle tissue was ground and solubilized in lysis buffer (Wuhan Boster Biological Technology, Ltd., Wuhan, China). Homogenates were centrifuged at $62,000 \mathrm{x}$ g at $4^{\circ} \mathrm{C}$ for $30 \mathrm{~min}$ and the supernatant was collected. The protein concentration of the samples was determined using a Bradford assay (11), with bovine serum albumin (Sigma-Aldrich, St. Louis, MO, USA) as the protein standard. Proteins in the homogenate fraction were separated by $8 \%$ SDS-PAGE and transferred to polyvinylidene difluoride membranes. Immunoblotting was subsequently performed with the following antibodies: Anti-TGF- $\beta 1$, anti-T $\beta$ RI, anti-T $\beta$ RII, anti-pSmad2, anti-pSmad3, anti-Smad2/3 and anti-collagen I. The antibodies anti-TGF- $\beta 1$ (cat. no. ab92486), anti-T $\beta$ RI (cat. no. ab31013), anti-T $\beta$ RII (cat. no. ab61213) and anti-collagen I (ab34710), all were purchased from Abcam (Cambridge, UK) and used at a dilution of 1:2,000, while anti-pSmad2 (cat. no. 3101), anti-pSmad3 (cat. no. 9520), anti-Smad2/3 (cat. no. 3102), and anti-GAPDH (cat. no. 2118), were purchased from Cell Signaling Technology, Inc. (Danvers, MA, USA) and used at a dilution of 1:1,000. Horseradish peroxidase (HRP)-conjugated Goat Anti-Rabbit IgG H\&L (cat. no. ab6721) was purchased from Abcam, and used as the secondary antibody at a dilution of 1:2,000. Immunoreactive bands were visualized with an enhanced chemiluminescence kit (cat. no. ab5801; Abcam). The bands on the western blots were scanned and the intensity of the bands was determined from optical density measurements using Gel-Pro Analyzer 4.0 software (Media Cybernetics, Inc, Rockville, MD, USA).

Statistical analysis. Data were analyzed with the SPSS version 20.0 software (IBM Corp., Armonk, NY, USA). The results were presented as the mean \pm standard deviation. Differences between each group were analyzed with two-way analysis of variance. $\mathrm{P}<0.05$ was considered to indicate a statistically significant difference.

\section{Results}

Radiography. X-ray scans of the femur were performed, and representative images at various time points are shown in Fig. 1. The scans provided evidence of osteomyelitis on post-wounding days 14 and 28 in group B. A minimal periosteal bone response was visible at day 7, while signs of infected tissue cortical bone response were observed at day 14. Evident diaphyseal osteolysis and calcification were present at day 28 (Fig. 1), which are characteristics of chronic osteomyelitis, thus indicating that the model of osteomyelitis was successfully established.

$H \& E$ staining. The results of $\mathrm{H} \& \mathrm{E}$ staining indicated a smaller number of fibroblasts in group A, while the muscle fibers were well-arranged (Fig. 2A). In group B, H\&E staining of muscle tissue revealed a high degree of chronic inflammation, an increased number of blood vessels and disarranged collagen tissue with a vortex-like distribution (Fig. 2B). By contrast, in group $\mathrm{C}, \mathrm{H} \& \mathrm{E}$ staining of decorin-treated tissues demonstrated a gradual reduction in blood vessels, fibroblasts and 
A

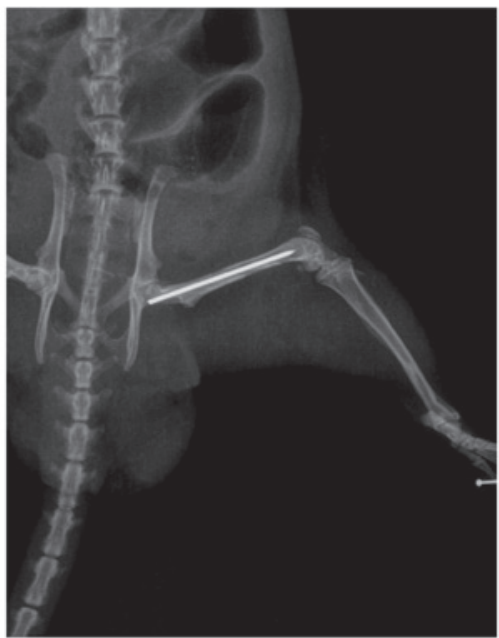

C

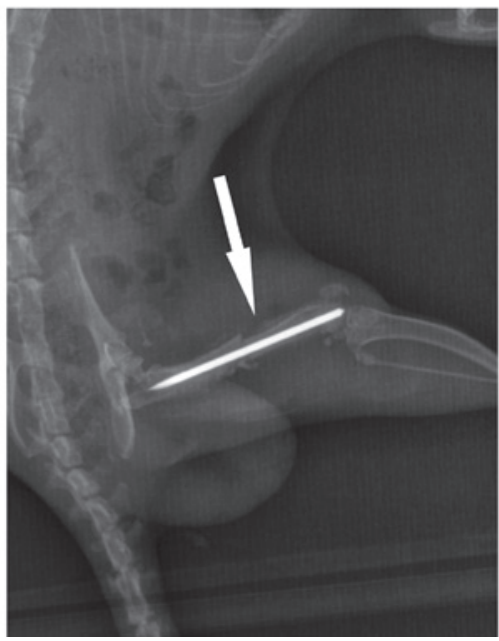

B

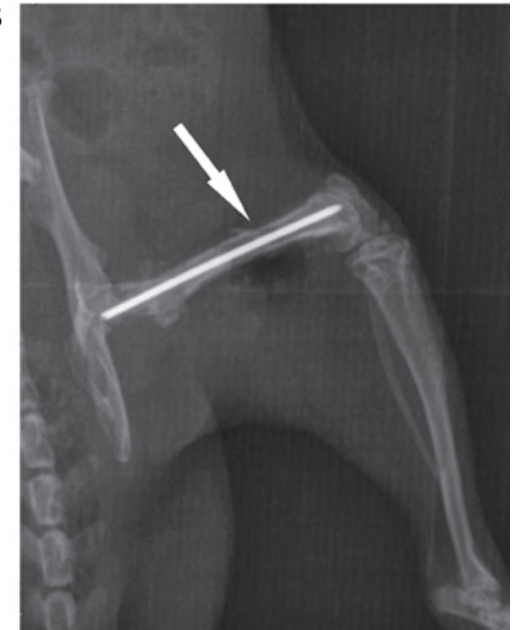

D

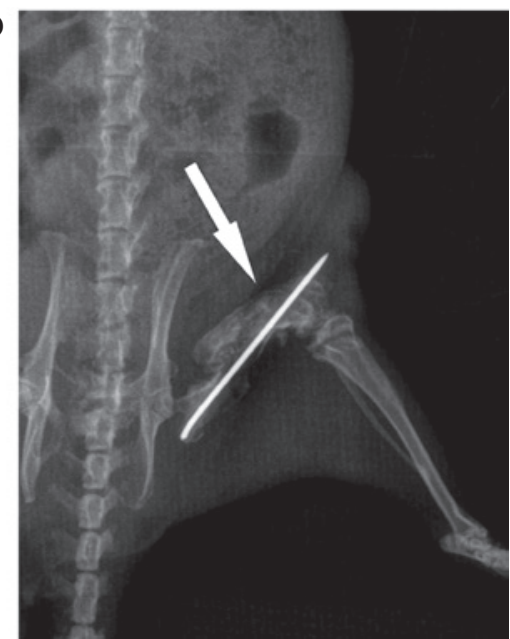

Figure 1. X-ray images during follow-up in the osteomyelitis group. (A) At 1 day after wounding, the X-ray image showed abnormal morphology of the bone tissue with correct implant placement. (B) A minimal periosteal bone response was observed 1 week after surgery. (C) Signs of osteomyelitis with periosteal reactivity were observed at 2 weeks after surgery. (D) Diaphyseal osteolysis and calcification were observed at week 4 after surgery.

collagen fibers, as well as a reduced number and ordered arrangement of collagen fibers (Fig. 2C).

Masson's trichrome staining. Under Masson's trichrome staining, skeletal muscle fibers appeared red and cell nuclei appeared dark blue, while collagen fibers appeared light blue. Progressively accumulation of collagen was considered as a hallmark of tissue fibrosis. In group A, muscle fibers were regularly arranged with presence of few detached collagen deposits among muscle fibers (Fig. 3A). In group B, a large amount of blue proliferating collagen around atrophic muscle fibers was observed, indicating fibrosis alterations in the skeletal muscle (Fig. 3B). Following treatment with decorin, collagen production was significantly reduced in group $\mathrm{C}$ as compared with that observed in group B (Fig. 3C).

Expression of TGF- $\beta 1$ and activation of the TGF- $\beta 1 /$ Smad signaling pathway. To determine whether activation of TGF- $\beta 1$ serves a role in the fibrosis typically observed in osteomyelitis, the expression of TGF- $\beta 1$ and activation of the TGF- $\beta 1 /$ Smad signaling pathway in the rat osteomyelitis model were investigated. As shown in Fig. 4, TGF- $\beta 1$, T $\beta$ RI, $\mathrm{T} \beta \mathrm{RII}, \mathrm{pSmad} 2$ and $\mathrm{pSmad} 3$ proteins in the control group were expressed at similar levels at different time points
(1, 2 and 4 weeks after wounding). In group B, the expression levels of TGF- $\beta 1, T \beta R I, T \beta R I I, p S m a d 2$ and $p S m a d 3$ were significantly increased at days 14 and 28 after surgery, when compared with the control group for each protein $(\mathrm{P}<0.05)$. This suggests that the TGF- $\beta 1 /$ Smad signaling pathway served an important role in the fibrosis observed in the osteomyelitis model group. Furthermore, it indicates that scar formation occurred at days 14 and 28 after bacterial inoculation.

Effect of decorin in blocking muscle scar formation in osteomyelitis model. In order to further determine whether decorin is able to reduce muscle scarring in osteomyelitis, the study examined the expression levels of TGF- $\beta 1$, pSmad2, pSmad3 and collagen I at post-wounding days 14 and 28. As shown in Fig. 5, TGF- $\beta 1$, pSmad 2 , pSmad 3 and collagen I expression levels in the decorin-treated group were higher than those in the control group at days 14 and $28(\mathrm{P}<0.05)$. However, decorin injection resulted in significant downregulation of these proteins at days 14 and 28, when compared with the levels in the osteomyelitis group $(\mathrm{P}<0.05)$. This suggests that decorin is able to reduce the formation of muscle scarring in osteomyelitis through inhibition of the TGF- $\beta 1$ signaling pathway. 
A

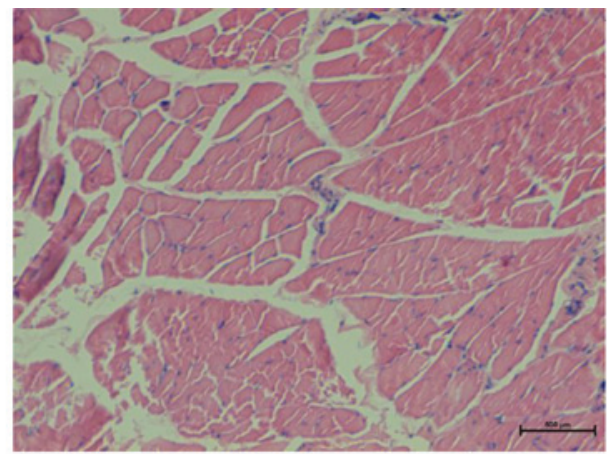

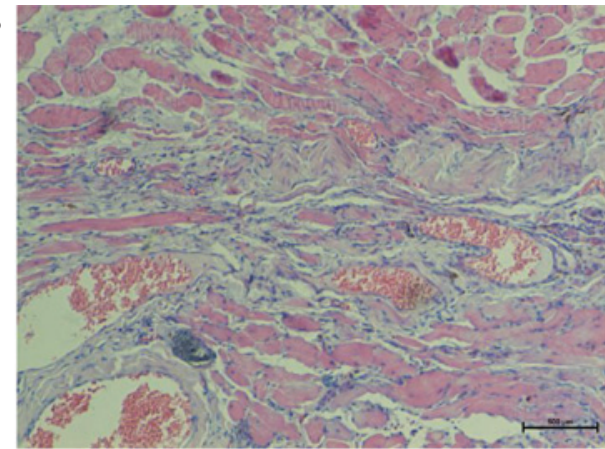

C

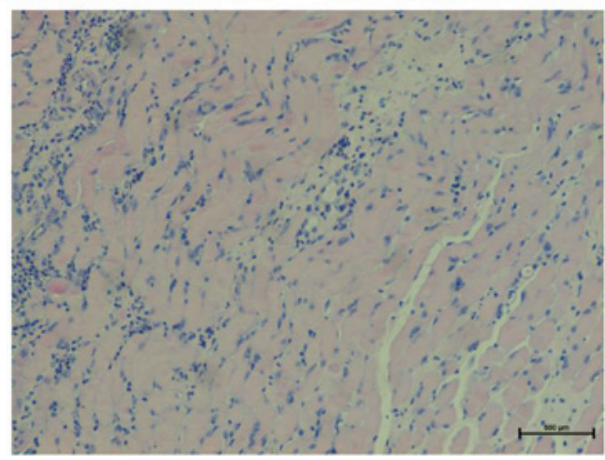

Figure 2. Hematoxylin-eosin staining of representative tissue samples collected at 4 weeks after surgery in the (A) control, (B) osteomyelitis model and (C) decorin-treated groups (magnification, x100). The control group showed well-arranged muscle fibers and few microvessels. In the osteomyelitis group, tissue was characterized by evident hyperplasia, with an increased number of microvessels, chronic inflammation, and irregularly-arranged nodular, circular or whorled muscle fibers. The decorin-treated group showed a gradual reduction in blood vessels and chronic inflammation, with well-arranged muscle fibers.

A

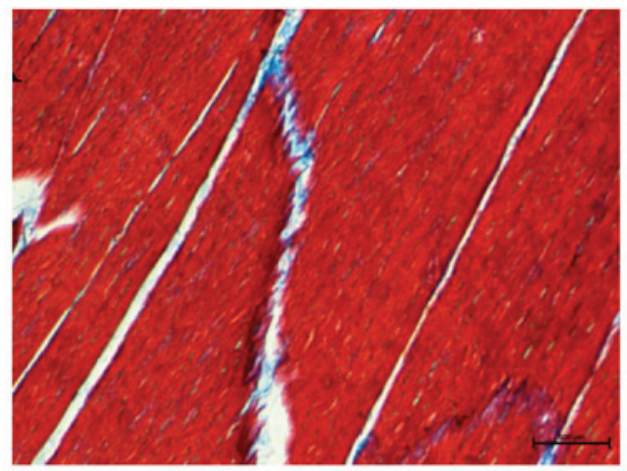

B

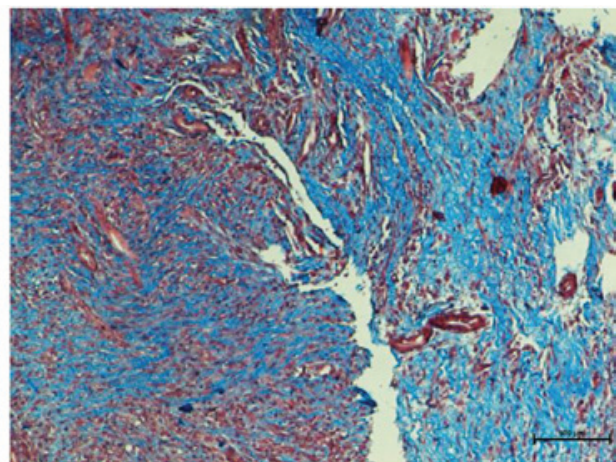

C

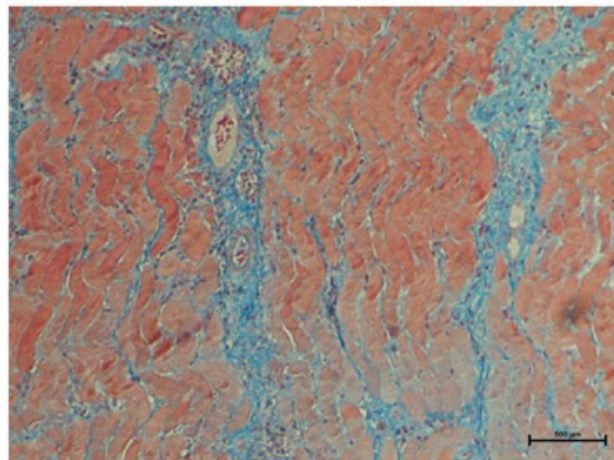

Figure 3. Masson's trichrome staining representative tissue samples in different groups (magnification, x200). (A) In the control group, red muscle fibers were regularly arranged with presence of few detached collagen deposits. (B) In the osteomyelitis group, atrophic muscle fibers were irregularly arranged and increased blue collagen areas was present. (C) In the decorin-treated group, muscle fibers were relatively regular and blue areas of collagen deposition were reduced.

\section{Discussion}

Chronic osteomyelitis is an infection of the bone, which frequently results in unusual formation of hypertrophic scars in the surrounding soft tissue (12). Treatment for hypertrophic scarring in osteomyelitis is challenging, and a good understanding of underlying molecular processes is essential for the optimal treatment of such hypertrophic scars (13). 


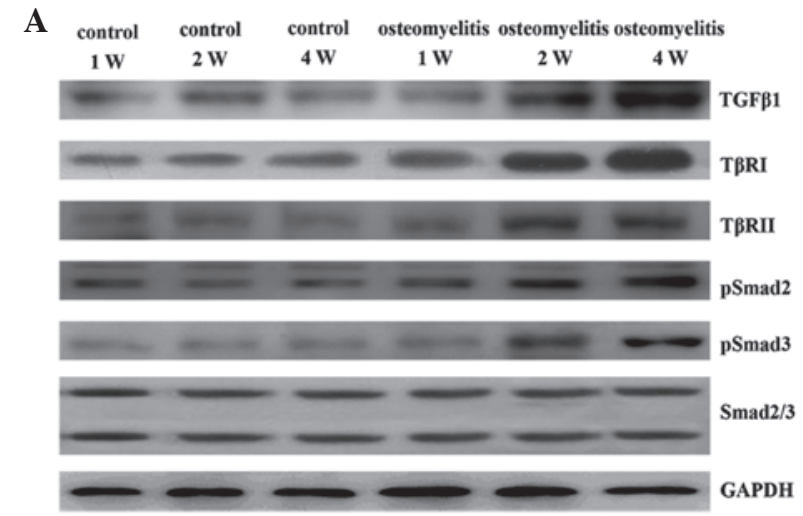

B
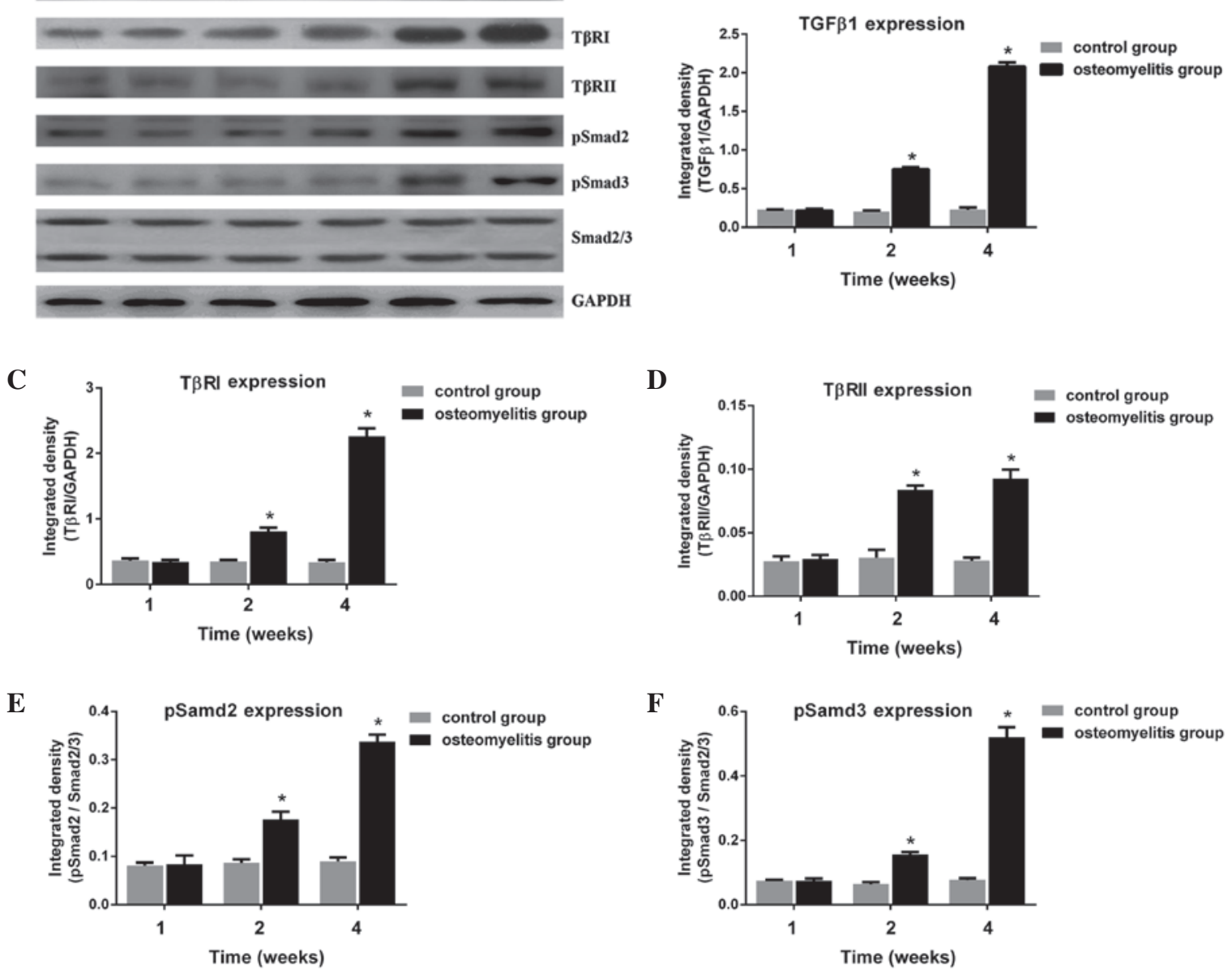

Figure 4. (A) Western blot analysis in the control and osteomyelitis groups at weeks (W) 1,2 and 4 after surgery. The expression levels of (B) TGF- $\beta 1$, (C) T $\beta R I$, (D) TRRII, (E) pSmad2 and (F) pSmad3 are shown as the integrated density against GAPDH and Smad2/3. "P $<0.05$ vs. the corresponding osteomyelitis group. TGF, transforming growth factor; T $\beta R$, TGF- $\beta$ receptor.

Collagen is the predominant component of scars, which are elaborated by fibroblasts. The pathogenesis of scar formation is regulated through a balance between collagen synthesis and degradation (14). Collagen is a molecule composed of three polypeptide chains. Cross-links between staggered collagen molecules provide stability and strength to the extracellular matrix (15). The scar tissue is characterized by the overproduction and deposition of type I and III collagen by fibroblasts (16).

Various cytokines are important components in the process of collagen synthesis. Among these, TGF- $\beta$ is a collagen synthesis stimulator and the most potent inducers of fibrosis (17). The TGF- $\beta$ family members control cell proliferation, differentiation, apoptosis, migration and extracellular matrix production (18). There are three isoforms of TGF- $\beta$ expressed in mammalian tissues: TGF- $\beta 1$ and TGF- $\beta 2$ have been identified as serving major role in tissue fibrosis $(19,20)$, while TGF- $\beta 3$ has been shown to reduce scar formation (21). All three isoforms are considered to function through the TGF- $\beta$ signaling cascade pathway in wound healing and abnormal scar formation. TGF- $\beta$ signaling is initiated by its binding to membrane receptor type II (namely T $\beta R I I)$. Subsequent to binding of the ligand, T $\beta$ RII forms a serine/threonine kinase complex with T $\beta R I$, which leads to the subsequent intracellular Smad cascade. This initiates the phosphorylation of Smad2 and Smad 3 by TGF- $\beta$ receptors, resulting in the formation of complexes with Smad4 (22). The Smad2-Smad3-Smad4 complex then translocates into the nucleus as a transcription factor and binds to promoters of type I, II and VII collagen genes in order to initiate collagen synthesis $(23,24)$. An increasing number of studies have strongly suggested a correlation among TGF- $\beta$ receptor, Smad activity, and fibroblast overproliferation and collagen production that leads to abnormal scar formation and pathologic fibrogenesis $(25,26)$.

Due to the close association between TGF- $\beta$ signaling and the production of collagen, blocking the TGF- $\beta$ signaling pathway may prevent the formation of scars. There are various negative regulators of TGF- $\beta$ signaling, including decorin, SnoN and Ski (27). Decorin is a small leucine-rich proteoglycan that serves an important role in regulating a myriad of functions in the extracellular matrix. It consists of a $40 \mathrm{kD}$ core protein and a glycosaminoglycan side chain $(28,29)$. Decorin has been shown to bind TGF- $\beta$ via the core protein and not the side chain, leading to an anti-fibrotic response (30). Previous studies have demonstrated that decorin has a downregulatory effect on TGF- $\beta$ production and collagen synthesis 
A

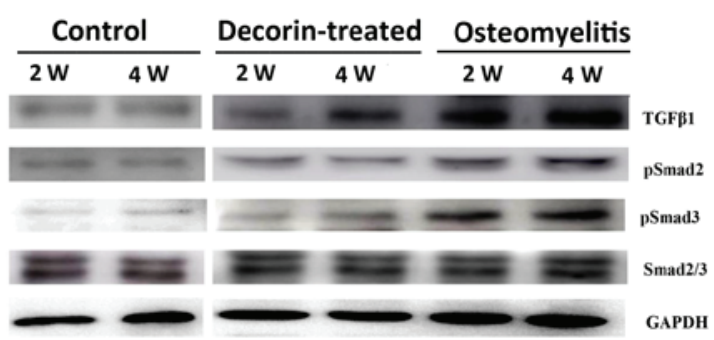

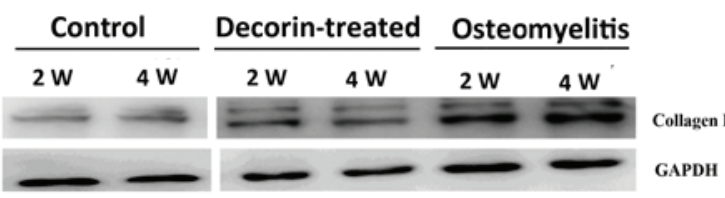

C

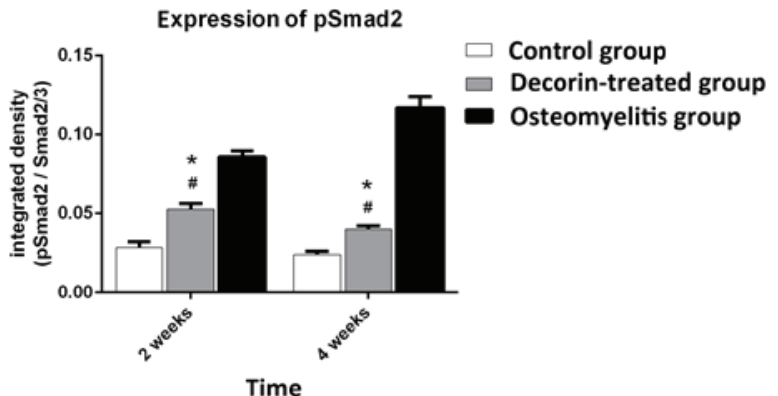

D
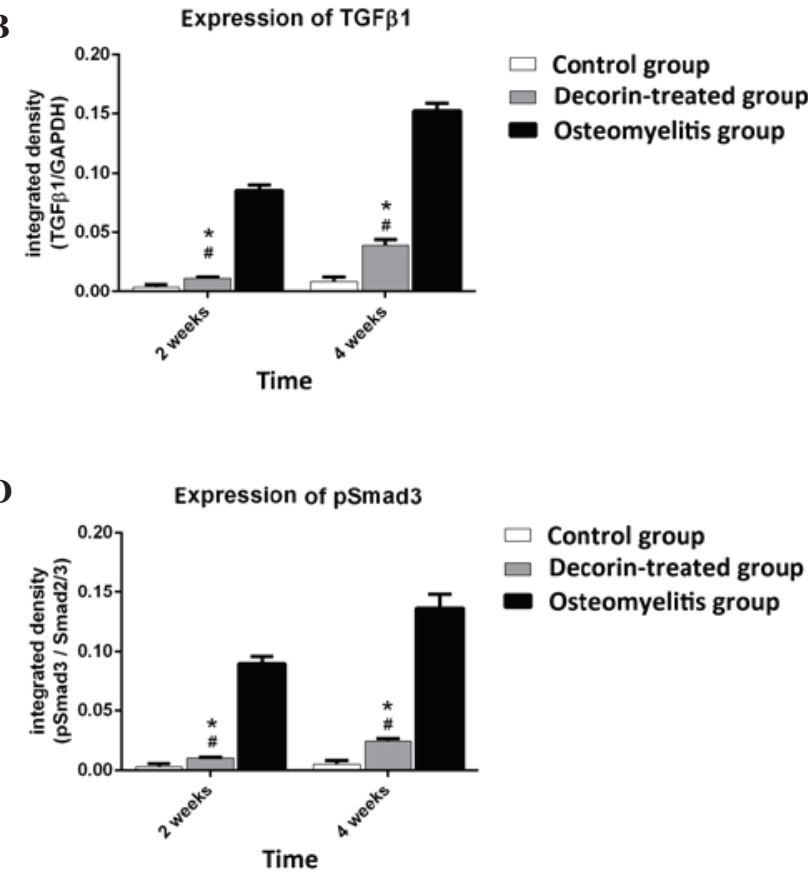

$\square$ Control group $\square$ Decorin-treated group - Osteomyelitis group

Figure 5. (A) Immunoblotting analysis in the control, osteomyelitis and decorin-treated groups at weeks (W) 2 and 4 after surgery. The expression levels of (B) TGF- $\beta 1$, (C) pSmad2, (D) pSmad3 and (E) collagen I are shown as the integrated density against GAPDH or Smad2/3. "P<0.05 vs. the corresponding control group; ${ }^{*} \mathrm{P}<0.05$ vs. the corresponding osteomyelitis group. TGF, transforming growth factor; T $\beta \mathrm{R}, \mathrm{TGF}-\beta$ receptor.

in hypertrophic scar fibroblasts in vitro $(31,32)$. In vivo experiments, in which decorin was injected or synthesized from an expression vector, revealed that this proteoglycan had a beneficial anti-fibrotic effect (33). The role of decorin in anti-fibrotic activity and differentiation in skeletal muscle by binding TGF- $\beta$ and myostatin has been well established $(34,35)$. Furthermore, the number of fibroblasts in decorin-deficient mice has been shown to increase by two-fold as compared with that in wild-type mice (36).

In the present study, an animal model mimicking the biological development of osteomyelitis was used to determine the effect of decorin in inhibiting scar formation in osteomyelitis. The study initially examined the alterations in the expression levels of TGF- $\beta 1$ to determine whether the activation of TGF- $\beta 1$ is involved in the fibrosis observed in osteomyelitis. The results revealed an increased expression of TGF- $\beta 1$ in osteomyelitis. In order to better understand the possible mechanisms of fibrosis, the activities of T $\beta$ RI, T $\beta$ RII, Smad 2 and Smad3 were examined, which are downstream targets of TGF- $\beta 1$ mediated signaling. The results demonstrated the involvement of TGF- $\beta 1$ signaling in the transmission of fibrosis signals in osteomyelitis. To further investigate the effect and mechanism of decorin on reducing scar formation, the degree of fibrosis in the surrounding tissue was investigated by Masson's trichrome staining, while the activation of TGF- $\beta 1$ signaling was examined, in the decorin-treatment group. Following decorin treatment, fibrotic changes in the surrounding tissue were reduced and the activation of TGF- $\beta 1$ signaling significantly decreased. This indicated that one of the mechanisms through which decorin decreased scarring in osteomyelitis was by inhibiting the TGF- $\beta 1$ signaling pathway.

However, there are certain limitations to this study. Although animals received the same dose of MSSA for osteomyelitis or decorin for treatment, intersubject variability of the animals may have potentially affected the degree of scarring. In addition, the anti-fibrotic effect of decorin in muscle tissue seemed to be dose-dependent and time-dependent. Fukushima et al (37) revealed that an injection of $50 \mu \mathrm{g}$ decorin at 10 and 15 days after injury significantly decreased the amount of fibrosis. In the present study, three injections of decorin at a dose of $50 \mu \mathrm{g}$ each time were performed, and a significant reduction in fibrosis was observed. Future studies should investigate the dose and time responses of the anti-fibrotic effect of decorin, as well as the underlying molecular mechanism in vitro. 
In conclusion, the present study demonstrated the involvement of the TGF- $\beta 1$ cascade in scar formation in osteomyelitis. Decorin was shown to reduce type I collagen expression in osteomyelitis rats in vivo through the inhibition of TGF- $\beta 1$ signaling pathway. Therefore, suppressing TGF- $\beta 1$ activity by decorin treatment may be a feasible method to limit the negative effects of scar formation and promote muscle functional recovery in osteomyelitis. The use of gene therapy and cell therapy procedures to deliver a high level of decorin expression to reduce scar in osteomyelitis will be further investigated.

\section{References}

1. Lazzarini L, De Lalla F and Mader JT: Long bone osteomyelitis Curr Infect Dis Rep 4: 439-445, 2002.

2. Eid AJ and Berbari EF: Osteomyelitis: Review of pathophysiology, diagnostic modalities and therapeutic options. J Med Liban 60: 51-60, 2012.

3. Papakostidis C, Bhandari M and Giannoudis PV: Distraction osteogenesis in the treatment of long bone defects of the lower limbs: Effectiveness, complications and clinical results a systematic review and meta-analysis. Bone Joint J 95-B $1673-1680,2013$

4. Lowenberg DW, Buntic RF, Buncke GM and Parrett BM: Long-term results and costs of muscle flap coverage with Ilizarov bone transport in lower limb salvage. J Orthop Trauma 27: 576-581, 2013

5. Xiao Z and Xi C: Hepatocyte growth factor reduces hypertrophy of skin scar: In vivo study. Adv Skin Wound Care 26: 266-270, 2013.

6. Sriram S, Robinson P, Pi L, Lewin AS and Schultz G: Triple combination of siRNAs targeting TGF $\beta 1$, TGF $\beta R 2$, and CTGF enhances reduction of collagen I and smooth muscle actin in corneal fibroblasts. Invest Ophthalmol Vis Sci. 54: 8214-8223, 2013.

7. Cutroneo KR, White SL, Phan SH and Ehrlich HP: Therapies for bleomycin induced lung fibrosis through regulation of TGF-betal induced collagen gene expression. J Cell Physiol 211: 585-589, 2007.

8. Zhang Z, Li XJ, Liu Y, Zhang X, Li YY and Xu WS: Recombinant human decorin inhibits cell proliferation and downregulates TGF-betal production in hypertrophic scar fibroblasts. Burns 33: 634-641, 2007.

9. Inanmaz ME, Uslu M, Isik C, Kaya E, Tas T and Bayram R: Extracorporeal shockwave increases the effectiveness of systemic antibiotic treatment in implant-related chronic osteomyelitis: Experimental study in a rat model. J Orthop Res 32: 752-756, 2014

10. Kishor C, Mishra RR, Saraf SK, Kumar M, Srivastav AK and Nath G: Phage therapy of staphylococcal chronic osteomyelitis in experimental animal model. Indian J Med Res 143: 87-94, 2016.

11. Cheng Y, Wei H, Sun R, Tian Z and Zheng X: Rapid method for protein quantitation by Bradford assay after elimination of the interference of polysorbate 80. Anal Biochem 494: 37-39, 2016.

12. Orimolade EA, Olabanji JK, Oladele AO and Yusuf MB: Chronic osteomyelitis in the lower extremity predisposing to the unusual formation of keloids. Singapore Med J 52: e190-e193, 2011.

13. Wolfram D, Tzankov A, Pülzl P and Piza-Katzer H: Hypertrophic scars and keloids - a review of their pathophysiology, risk factors, and therapeutic management. Dermatol Surg 35: 171-181, 2009.

14. Jia S, Zhao Y, Law M, Galiano R and Mustoe TA: The effects of collagenase ointment on the prevention of hypertrophic scarring in a rabbit ear scarring model: A pilot study. Wounds 23: 160-165, 2011.

15. Boudko SP, Engel J and Bächinger HP: The crucial role of trimerization domains in collagen folding. Int J Biochem Cell Biol 44: 21-32, 2012.

16. Bhogal RK, Stoica CM, McGaha TL and Bona CA: Molecular aspects of regulation of collagen gene expression in fibrosis. J Clin Immunol 25: 592-603, 2005.

17. Gharaee-Kermani M, Hu B, Phan SH and Gyetko MR: Recent advances in molecular targets and treatment of idiopathic pulmonary fibrosis: Focus on TGFbeta signaling and the myofibroblast. Curr Med Chem 16: 1400-1417, 2009.
18. Whitman M: Smads and early developmental signaling by the TGFbeta superfamily. Genes 12: 2445-2462, 1998.

19. Joyce ME, Roberts AB, Sporn MB and Bolander ME: Transforming growth factor-beta and the initiation of chondrogenesis and osteogenesis in the rat femur. J Cell Biol 110: 2195-2207, 1990

20. Hou XH, Cao B, Liu HQ, Wang YZ, Bai SF and Chen H: Effects of osthole on apoptosis and TGF-betal of hypertrophic scar fibroblasts. J Asian Nat Prod Res 11: 663-669, 2009.

21. Waddington SN, Crossley R, Sheard V, Howe SJ, Buckley SM, Coughlan L, Gilham DE, Hawkins RE and McKay TR: Gene delivery of a mutant TGF $\beta 3$ reduces markers of scar tissue formation after cutaneous wounding. Mol Ther 18: 2104-2111, 2010.

22. Massagué J: TGFbeta signaling: Receptors, transducers and Mad proteins. Cell 85: 947-950, 1996.

23. Peltonen J, Kähäri L, Jaakkola S, Kähäri VM, Varga J, Uitto J and Jimenez SA: Evaluation of transforming growth factor beta and type I procollagen gene expression in fibrotic skin diseases by in situ hybridization. J Invest Dermatol 94: 365-371, 1990.

24. Wang X, Smith P, Pu LL, Kim YJ, Ko F and Robson MC: Exogenous transforming growth factor beta(2) modulates collagen I and collagen III synthesis in proliferative scar xenografts in nude rats. J Surg Res 87: 194-200, 1999.

25. Fan C, Dong Y, Xie Y, Su Y, Zhang X, Leavesley D and Upton Z: Shikonin reduces TGF- $\beta 1$-induced collagen production and contraction in hypertrophic scar-derived human skin fibroblasts. Int J Mol Med 36: 985-991, 2015.

26. Bai X, He T, Liu J, Wang Y, Fan L, Tao K, Shi J, Tang C, Su L and $\mathrm{Hu} \mathrm{D}$ : Loureirin B inhibits fibroblast proliferation and extracellular matrix deposition in hypertrophic scar via TGF- $\beta /$ Smad pathway. Exp Dermatol 24: 355-360, 2015.

27. Deheuninck J and Luo K: Ski and SnoN, potent negative regulators of TGF-beta signaling. Cell Res 19: 47-57, 2009.

28. Iozzo RV: Matrix proteoglycans: From molecular design to cellular function. Annu Rev Biochem 67: 609-652, 1998.

29. Ameye L and Young MF: Mice deficient in small leucine-rich proteoglycans: Novel in vivo models for osteoporosis, osteoarthritis, Ehlers-Danlos syndrome, muscular dystrophy and corneal diseases. Glycobiology 12: 107R-116R, 2002.

30. Miura T, Kishioka Y, Wakamatsu J, Hattori A, Hennebry A, Berry CJ, Sharma M, Kambadur R and Nishimura T: Decorin binds myostatin and modulates its activity to muscle cells. Biochem Biophys Res Commun 340: 675-680, 2006.

31. Ahmed Z, Bansal D, Tizzard K, Surey S, Esmaeili M, Gonzalez AM, Berry M and Logan A: Decorin blocks scarring and cystic cavitation in acute and induces scar dissolution in chronic spinal cord wounds. Neurobiol Dis 64: 163-176, 2014.

32. Honardoust D, Varkey M, Hori K, Ding J, Shankowsky HA and Tredget EE: Small leucine-rich proteoglycans, decorin and fibromodulin, are reduced in postburn hypertrophic scar. Wound Repair Regen 19: 368-378, 2011.

33. Kolb M, Margetts PJ, Sime PJ and Gauldie J: Proteoglycans decorin and biglycan differentially modulate TGF-beta-mediated fibrotic responses in the lung. Am J Physiol Lung Cell Mol Physiol 280: L1327-L1334, 2001

34. Zhu J, Li Y, Shen W, Qiao C, Ambrosio F, Lavasani M, Nozaki M, Branca MF and Huard J: Relationships between transforming growth factor-beta1, myostatin and decorin: Implications for skeletal muscle fibrosis. J Biol Chem 282: 25852-25863, 2007.

35. Kishioka Y, Thomas M, Wakamatsu J, Hattori A, Sharma M, Kambadur R and Nishimura T: Decorin enhances the proliferation and differentiation of myogenic cells through suppressing myostatin activity. J Cell Physiol 215: 856-867, 2008.

36. Hakkinen L, Strassburger S, Kähäri VM, Scott PG, Eichstetter I, Lozzo RV and Larjava H: A role for decorin in the structural organization of periodontal ligament. Lab Invest 80: 1869-1880, 2000.

37. Fukushima K, Badlani N, Usas A, Riano F, Fu F and Huard J: The use of an antifibrosis agent to improve muscle recovery after laceration. Am J Sports Med 29: 394-402, 2001. 